\title{
Avaliação radiográfica da silhueta cardíaca, pelo método VHS (Vertebral Heart Size), de quatis (Nasua nasua, Linnaeus 1766) jovens e adultos mantidos em cativeiro
}

\author{
Radiographic evaluation of the cardiac silluet using the VHS \\ method (Vertebral Heart Size) in young and adults coatis (Nasua \\ nasua, Linneaus 1766) living in captivity
}

\author{
Andresa de Cássia Martini1 ${ }^{*}$; Yara Silva Meireles ${ }^{1}$; Samuel Monzem²; \\ Luiz Paulo Vasconcelos'; ${ }^{2}$ Nívea Clarice Monteiro Rocha Turbino ${ }^{3}$; \\ Magyda Arabia Araji Dahroug 3 ; Daniela Farias 3 ; Pedro Brandini Néspoli ${ }^{4}$; \\ Gentil Ferreira Gonçalves ${ }^{4}$; Roberto Lopes de Souza ${ }^{4}$; \\ Luciana Dambrósio Guimarães ${ }^{4}$
}

\section{Resumo}

\begin{abstract}
O exame radiográfico do tórax é um método auxiliar de avaliação não invasiva, para acompanhamento da evolução de doenças cardíacas sugerindo prognósticos e orientando tratamentos. O objetivo desse trabalho foi avaliar a silhueta cardíaca de quatis adultos e jovens em relação ao número de vértebras torácicas (VHS), pelo método proposto por Buchanam e Buchele (1995) para pequenos animais. Avaliou-se 20 quatis, separados por faixa etária em grupo I (GI) sendo 8 animais de idade entre 4 e 5 meses e grupo II (GII) com 12 animais acima de 12 meses de idade. Realizou-se radiografias do tórax nas projeções ventrodorsal e laterolateral direita para determinação do eixo maior (L) e eixo menor (S), sendo a somatória de L e S o valor obtido de VHS, a relação profundidade/ largura (P/L) torácica foram obtidas e os resultados determinaram o tipo de conformação do tórax, onde resultados superiores a $1,25 \mathrm{~cm}$ denotaram tórax tipo profundo, de 0,75 a $1,25 \mathrm{~cm}$ tórax intermediário e resultados inferiores à $0,75 \mathrm{~cm}$ tórax largo. Observou-se que o coração está inserido entre o quarto e o sétimo par de costelas, o VHS médio de quatis adultos saudáveis foi de 9,36 $\pm 0,75$ e o de jovens $8,06 \pm 0,595$ unidades de vértebras e a conformação torácica predominante encontrada foi do tipo intermediária quando comparada aos cães. Os valores médios deste estudo servirão como base para interpretação do VHS na espécie, contudo um maior número animais pode ser necessário para determinação de limites morfológicos da silhueta cardíaca em quatis.
\end{abstract}

Palavras-chave: Quatis, silhueta cardíaca, vértebra

\footnotetext{
${ }^{1}$ Discentes de Mestrado do Programa de Pós-graduação em Ciências Veterinárias, CCPCVET, FAMEV, Universidade Federal de Mato Grosso UFMT, CAPES, Cuiabá, MT. E-mail: andresa.martini@hotmail.com; yarameireles@gmail.com

${ }^{2}$ Discentes de Especialização do Programa de Residência Uniprofissional em Medicina Veterinária, HOVET, UFMT, Cuiabá, MT. E-mail: s.monzem@hotmail.com; luiz_paulo@hotmail.com

${ }^{3}$ Médicas Veterinárias Autônomas, Cuiabá, MT. E-mail:nmrochaturbino@hotmail.com; magyda@gmail.com; daniela.farias.739@ facebook.com

${ }^{4}$ Profs. do Dept ${ }^{\circ}$ de Clínica Médica, CLIMEV/FAMEV, UFMT, Cuiabá, MT. E-mail: nespoli@ufmt.br; gentil.goncalves.jr@ facebook.com; rsouza@ufmt.br; ldguimaraes@ufmt.br

* Autor para correspondência
} 


\begin{abstract}
Radiographic examination of the toracic cavity is an usefull noninvasive method for assessment, monitoring the progress of heart disease, suggesting prognosis and guiding the treatment. The aim of this study was to evaluate the cardiac silhouette of young and adults coatis and evaluate its relationship to the number of thoracic vertebrae (VHS), the method proposed by Buchanam and Buchele (1995) for small animals. We evaluated a group of 20 coatis, divided by age: I (GI) and 8 animals aged between 4 and 5 months and group II (GII) with 12 animals over 12 months old. Based in chest radiographs and VD laterolateral right projections for determining the major axis $(\mathrm{L})$ and short axis $(\mathrm{Y})$ being the sum of $\mathrm{L}$ and $\mathrm{S}$ is the value obtained by ESR, the relative depth/width (D / L) chest were obtained and the results determined the type of conformation of the thorax, which results greater than $1.25 \mathrm{~cm}$ denote chest type deep, 0.75 to $1.25 \mathrm{~cm}$ chest intermediate and inferior results will $0.75 \mathrm{~cm}$ wide chest. It was observed that the heart is alocated between the fourth and seventh pair of ribs, VHS average coatis healthy adults was $9.36 \pm 0.75$ and $8.06 \pm 0$ youth, 595 units thoracic vertebrae and the predominant conformation found was of intermediate type when compared to dogs. The mean values in this study serve as a basis for interpretation of the VHS type, however, a larger number may be required animals to determine the physiological limits of the cardiac silhouette in coati.
\end{abstract}

Key words: Coatis, cardiac silhouette, vertebra

\section{Introdução}

O quati (Nasua nausa) pertence ao filo Chordata, classe Mammalia, ordem Carnívora e à família Procyonidae, sendo um animal onívoro, de hábito diurno e encontrado amplamente na América do sul, ocupando quase todas as regiões do Brasil (TEIXEIRA; AMBRÓSIO 2007). São poucos os relatos que indiquem doenças cardiovasculares em quatis, no entanto são animais que envelhecem em condições de cativeiro, tornando-se potencialmente portadores de doenças cardíacas. No Pantanal sul, a ocorrência de Trypanossoma cruzi em quatis tem sido bem documentada, mostrando que essa espécie de mamífero representa um importante papel no sistema reservatório de $T$. cruzi, contribuindo com a prevalência da doença de Chagas, sendo essa um sério problema de saúde pública na América Latina. (SILVA; SOUZA-LIMA, 2004). Atualmente a falta de conhecimento da anatomia radiográfica de animais silvestres ainda é um dos elementos que dificultam a interpretação dos exames desses animais. Mesmo quando se procura agrupar os animais por similaridade dentro das classes (aves, répteis e mamíferos), persiste ainda uma incalculável variação anatômica entre os membros de cada grupo (FONSECA, 2007) necessitando estabelecer parâmetros para o diagnóstico radiológico.
O diagnóstico por imagem apresenta grandes avanços na área de cardiologia, pela possibilidade de avaliar e realizar exames do coração por métodos não invasivos (FRANDSON, 2005; RAMIREZ, 2005). Nesse contexto o exame radiográfico do tórax, eletrocardiograma e ecocardiograma, são ferramentas importantes e indispensáveis para o diagnóstico, prognóstico e orientação no tratamento das afecções cardiovasculares (ZANDVLIET, 2005; SUTER, 1987). Para evitarem-se imagens com distorções na silhueta cardíaca o animal deve ser posicionado corretamente (PATTON; FAULKNER, 1992; ROOT; BAHR, 1998; PINTO; IWASAKI, 2004). De acordo com Lamb et al (2000), exames radiográficos devem ser realizados no mínimo em duas projeções (lateral direita e ventrodorsal/ dorsoventral) ou para maior fidedignidade são realizadas três projeções (laterais direito e esquerdo e ventrodorsal/dorsoventral).

Análises subjetivas de exames radiográficos do coração servem para verificação de estruturas ali existentes, como os espaços intercostais, localização pulmonar, verificação de anormalidades, presença de protuberâncias, aumento no calibre dos vasos e projeções cardíacas para a área pulmonar (NAKAYAMA; NAKAYAMA; HAMLIN, 2001). Vários estudos foram realizados objetivando 
aumentar a acurácia e diminuir a subjetividade do estudo radiográfico da silhueta cardíaca (CARDOSO; CALUDINO; MELUSSI, 2011).

Um conhecimento da função cardíaca normal e dos vários mecanismos compensatórios é essencial para o acesso equilibrado e lógico à interpretação das radiografias cardíacas (SISSON; GROSMAN, 2000; CONSTANTINESCU, 2005). Contudo algumas anormalidades cardíacas podem ser sugeridas, dentre elas destacam-se: cardiomegalia, miocardiopatia dilatada, persistência de ducto arterioso e dirofilariose (VAN DEN BROOK; DARKE, 1987; STEPIEN; BENSON, FORREST, 1999; THRALL, 1998; HERRTAGE; DENNIS, 1999; SISSON; THOMAS; BONAGURA, 2004.; CONSTANTINESCU, 2005; FELIPPE, 2007).

Buchanan e Bucheler (1995) desenvolveram um método para identificar anatomicamente o coração e diferenciar os limites entre os tamanhos da silhueta cardíaca em relação às raças principalmente de cães. Fizeram comparações entre medidas da silhueta cardíaca em relação ao comprimento vertebral, ambos são mensuráveis em exames radiográficos do tórax, sabe-se que existe uma boa correlação entre o tamanho do coração e o comprimento do corpo vertebral. Avaliaram também a diferença entre cães com tórax largo ou profundo, determinando valores para os mesmos, no qual consideraram como tórax largo animais que obtivessem valores $\leq 0,75$ unidade de vértebra torácica e tórax profundo valores $\geq$ 1,25 , valores que estivessem entre $\leq 0,75$ e $\geq 1,25$ foram classificados como tórax intermediário. De acordo com Lamb et al. (2001) e Gulanber (2005), existem diferenças significativas entre os valores de VHS para as diferentes raças de cães, o que torna imprescindível a padronização desses valores para cada raça.

O objetivo deste estudo foi avaliar o tamanho do coração de quatis em relação ao número de vértebras torácicas (VHS), com intuito de padronizar valores para essa espécie.

\section{Materiais e Métodos}

Foram avaliados 20 quatis, machos e fêmeas, mantidos em cativeiro, clinicamente saudáveis ao exame físico, submetidos a jejum prévio de 12 horas para contenção química e realização do exame radiográfico analógico do tórax. Os animais foram separados de acordo com a faixa etária em dois (2) grupos. Participaram do grupo I (GI) oito animais jovens, com idade entre quatro (4) e cinco (5) meses e grupo II (GII), 12 animais adultos com idade acima de 12 meses. Os animais foram escolhidos ao acaso e realizou-se a contenção química com a associação de tiletamina e zolazepam (Zoletil ${ }^{\circledR} 50 \mathrm{mg}$ ), na dose de $7 \mathrm{mg} / \mathrm{Kg}$, aplicada por via intramuscular (IM), e obteve-se exame radiográfico do tórax nas projeções ventrodorsal e laterolateral direita.

Para avaliação do exame radiográfico do tórax realizou-se primeiramente uma observação subjetiva, por dois examinadores especialistas em radiodiagnóstico, pois o uso de opinião consensual como padrão de referência é bem estabelecido em radiologia (KIDO et al., 1994). Observaram o número total de costelas, localização do coração dentro do tórax considerando sua posição entre os espaços intercostais, profundidade do tórax (proporção do tórax ocupado pelo coração) e relação de contato com o esterno. Determinou-se as distâncias correspondentes ao eixo maior (L) e eixo menor (S) do coração, na projeção laterolateral, sendo L a distância correspondente desde o bordo ventral do centro do brônquio principal esquerdo ao contorno mais distante do ápice cardíaco, e $\mathrm{S}$ à linha perpendicular ao eixo maior na altura do terço central do coração (Figura 1). As medidas de L e S em centímetros foram transportadas para a coluna vertebral torácica na projeção laterolateral direita, iniciando a aferição junto à margem cranial da quarta vértebra torácica (T4), o resultado foi expresso em unidade de comprimento de vértebra. Após somatória dos valores de L e S obteve-se os valores de VHS.

Para determinar a profundidade do tórax $(\mathrm{P}) \mathrm{em}$ 
centímetros, utilizou-se a projeção laterolateral, essa foi medida a distância entre a borda cranial do processo xifóide, e a borda ventral da coluna junto à oitava vértebra torácica por meio de linha perpendicular à coluna vertebral. Para determinação da largura do tórax (L) utilizou-se a projeção ventrodorsal, medindo em centímetros a distância entre as bordas mediais da oitava costela junto à sua curvatura lateral. Á exemplo de Buchaman e Bucheler (1995), o valor de (P) foi dividido pelo valor de (L), sendo considerado tórax profundo resultados superiores a $1,25 \mathrm{~cm}$, tórax largo resultados inferiores á $0,75 \mathrm{~cm}$ e intermediário quando o valor de $\mathrm{P} / \mathrm{L}$ estivesse entre esses limites.

Para comparação entre os valores médios de EL, ES, VHS, P, L e P/L do GI e do GII realizouse o teste de análise de variância (ANOVA) com software SAEG 5-0.

Figura 1. Ilustração radiográfica das medidas do eixo maior (EL) e eixo menor (EC) utilizados para mensuração do tamanho do coração em relação à unidade de vértebra torácica (VHS), ECv medida eixo curto convertida em número de vértebras ; ELv medida do eixo longo convertida em número de vértebras; Xv número total de vértebras de quatis (Nasua nasua) mantidos em cativeiro. Fonte: Arquivo Pessoal.

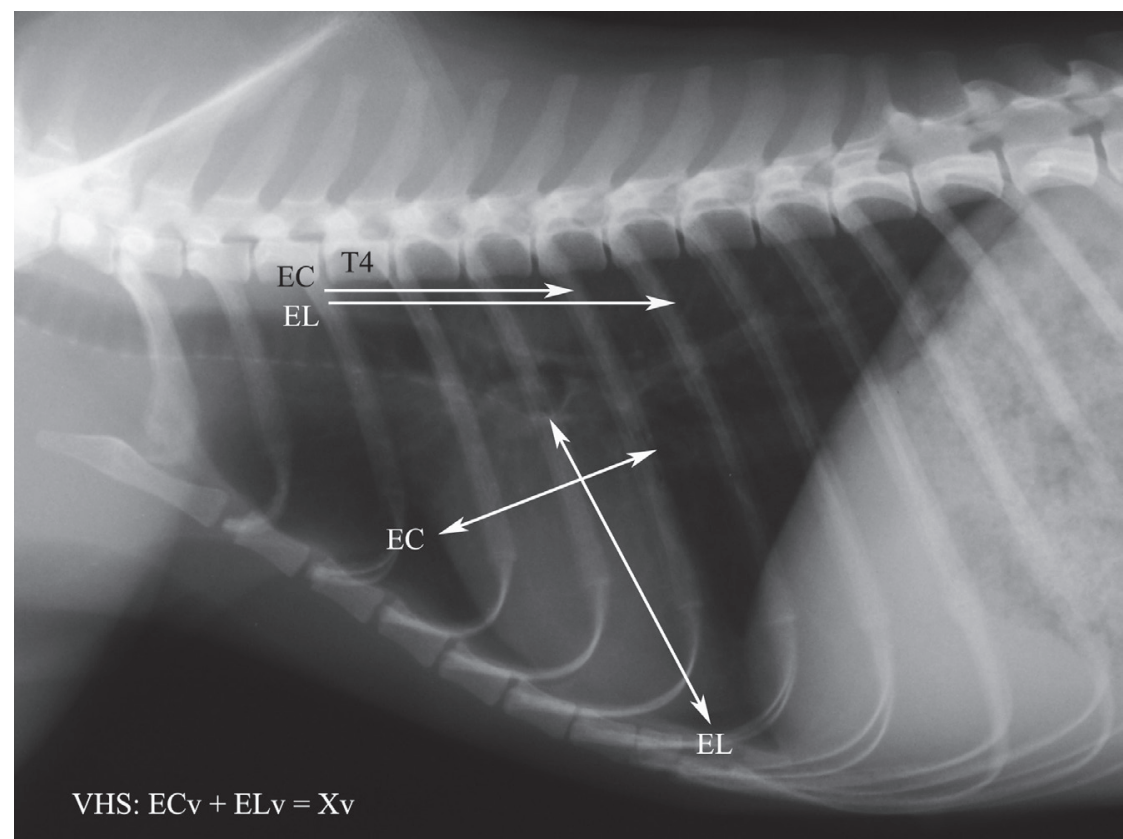

Fonte: Elaboração dos autores.

\section{Resultados}

$\mathrm{Na}$ avaliação subjetiva do exame radiográfico do tórax de quatis (Nasua nasua), observou-se um total de 15 pares de costelas, sendo que o coração estava inserido entre o $4^{\circ}$ e o $7^{\circ}$ pares, em todos animais examinados.

Pelo método de VHS, observou-se que os valores da mensuração do eixo maior $(\mathrm{L})$ variaram entre 2,4 a $3,4 \mathrm{~cm}$ no GI e 4,6 a $6,6 \mathrm{~cm}$ no GII, com média de 2,97( $\pm 0,369)$ e 5,37( $\pm 0,58) \mathrm{cm}$, respectivamente. $\mathrm{O}$ eixo menor variou de 1,6 a $2,1 \mathrm{~cm}$ no GI e de 2,9 a $4,8 \mathrm{~cm}$ no GII, com média de $1,86( \pm 0,206)$ e $3,74( \pm 0,62) \mathrm{cm}$ respectivamente. O VHS variou de 7,6 a $9,4 \mathrm{~cm}$ nos animais do GI e de 6,6 a $9,6 \mathrm{~cm}$ nos animais do GII com média de $8,06( \pm 0,595)$ e $9,36( \pm 0,75)$ unidades de vértebra, respectivamente.

A largura do tórax variou de 3,5 a 4,3cm no GI e 
de 7,1 a $8,2 \mathrm{~cm}$ no GII, com média de $4,76( \pm 0,417)$ e $7,77( \pm 0,68) \mathrm{cm}$ respectivamente. A profundidade do tórax variou de 4,6 a 5,5cm no GI e de 6,6 a 9,5cm no GII, com média de $4,12( \pm 0,452)$ e $8,13( \pm 0,98)$ $\mathrm{cm}$, respectivamente. A relação $\mathrm{P} / \mathrm{L}$ variou de 0,71 a $1,21 \mathrm{~cm}$, nos animais do GI e de 0,88 e $1,23 \mathrm{~cm}$ nos animais do GII com média de $0,87( \pm 0,153)$ e $1,05( \pm 0,11) \mathrm{cm}$ respectivamente.

Houve diferença estatística entre os valores das variáveis do GI e GII, sendo maior em GII. Os valores médios e desvio padrão das variáveis acima citadas estão relacionados na tabela 1 .

Tabela 1. Valores médios e desvio padrão das variáveis utilizadas para mensuração do tamanho do coração em relação à unidade de vértebras torácicas de quatis filhotes (GI) e adultos(GII) mantidos em cativeiro.

\begin{tabular}{lcc}
\hline \multirow{2}{*}{ VARIÁVEIS } & \multicolumn{2}{c}{ MÉDIA \pm DESVIO PADRÃO } \\
\cline { 2 - 3 } & GI $(\mathrm{n}=8)$ & GII $(\mathrm{n}=12)$ \\
\hline Eixo maior $(\mathrm{cm})$ & $2,97 \pm 0,369^{\mathrm{b}}$ & $5,37 \pm 0,58^{\mathrm{a}}$ \\
Eixo menor $(\mathrm{cm})$ & $1,86 \pm 0,206^{\mathrm{b}}$ & $3,74 \pm 0,62^{\mathrm{a}}$ \\
VHS $^{1}$ (unidade de vértebra) & $8,06 \pm 0,595^{\mathrm{b}}$ & $9,36 \pm 0,75^{\mathrm{a}}$ \\
Largura do tórax $(\mathrm{cm})$ & $4,76 \pm 0,417^{\mathrm{b}}$ & $7,77 \pm 0,68^{\mathrm{a}}$ \\
Profundidade do tórax $(\mathrm{cm})$ & $4,12 \pm 0,452^{\mathrm{b}}$ & $8,13 \pm 0,98^{\mathrm{a}}$ \\
Profundidade / largura $(\mathrm{cm})$ & $0,87 \pm 0,153^{\mathrm{b}}$ & $1,05 \pm 0,11^{\mathrm{a}}$ \\
\hline
\end{tabular}

Médias seguidas da mesma letra não diferem entre si pelo método de Tuckey ao nível de 5\% de probabilidade.

${ }^{1}$ VHS: Vertebral Heart Size (Mensuração cardíaca em relação a unidade de vértebra torácica)

Fonte: Elaboração dos autores

\section{Discussão}

$\mathrm{Na}$ avaliação empírica das radiografias torácicas, pode-se observar que os animais do GI e GII apresentaram 15 pares de costela, corroborando as observações de Gregores (2006) que avaliou a anatomia topográfica de quatis. O coração esta inserido entre a quarta e a sétima vértebra torácica, o que difere de cães e gatos, que apresentam treze pares de costelas e o coração inserido entre a terceira e a sexta costelas, segundo Sisson e Grosman (2000).

O valor de VHS no GII $(9,36 \pm 0,75)$ foi inferior aos valores de cães adultos sadios da raça Poodle (10,12 $\pm 0,51)$, Greyhounds $(10,12 \pm 0,51)$, Schnauzer miniatura $(11,00)$, semelhante ao de Rottweilwers $(9,8 \pm 0,51)$ e superior ao de gatos sadios (7,5 $\pm 0,3)$, segundo Buchanan e Bucheler (1995), Pinto e Iwasaki (2004) e Marin et al. (2007). Cardoso, Caludino e Melussi (2011) em estudo com American pit bull terrier observaram pequenas variações nas medidas do VHS entre radiografias esquerdas e direitas, devido a diferentes fases do ciclo cardíaco e respiratório.

Deve-se ressaltar, entretanto, que os valores de VHS para avaliação do tamanho do coração podem ser afetados por vários fatores como variação individual em tamanho real, comprimento vertebral, estreitamentos do disco, bem como resultados variados obtidos pela avaliação de diferentes observadores. (HANSSON et al., 2005) e que os resultados obtidos com número pequeno de animais desse estudo, provavelmente não refletem com precisão os valores de VHS da espécie, contudo são bastante úteis como índices iniciais de referência.

Os valores da relação entre largura e profundidade em GII $(1,05 \pm 0,11)$ determinaram uma classificação de tórax do tipo intermediário em $100 \%$ dos animais, quando comparado aos valores determinados para cães, como citado por Buchanan e Bucheler (1995). No GI, 12,5\% (1) dos animais foram classificados como portadores de tórax largo, apresentando resultados inferiores à $0,75 \mathrm{~cm}$. Uma pequena variação na classificação do tipo de tórax 
também foi observada por Pinto e Iwasaki (2004) em poodles saudáveis e foi desconsiderada. A largura, profundidade e relação entre largura e profundidade do tórax, que apresentaram diferenças significativas entre os grupos, podem ser influenciadas pelo tamanho do animal e da fase respiratória que compreendia o momento do exame radiográfico.

Os resultados obtidos no presente estudo permitem concordar com Buchanan e Bucheler (1995) e Pinto e Iwasaki (2004) no que diz respeito às vantagens apresentadas pelo método de mensuração VHS para o diagnóstico de cardiomegalia e acompanhamento da evolução da doença cardíaca, pois é rápido, prático e de fácil reprodução. Contudo é fundamental que haja um acordo comum sobre como as medições devem ser realizadas entre os clínicos, e um monitoramento progressivo quando existirem alterações cardíacas deve ser realizado para o mesmo animal (HANSSON et al., 2005). O diagnóstico das doenças cardíacas não é baseado somente nos achados radiográficos, sendo necessário associar sinais clínicos, achados físicos e também exame eletrocardiográfico e ecocardiográfico como descrito por Lamb et al. (2000). Contudo neste estudo, os animais não apresentam diferenciação de raças, portanto resultados de VHS apresentamse mais homogêneos, quando não ocorrerem falhas técnicas na obtenção das imagens.

\section{Conclusão}

Conclui-se que os valores médios deste estudo servem como base para interpretação do VHS na espécie em questão, contudo faz-se necessária a avaliação de um número maior de animais para determinação de limites morfológicos.

\section{Agradecimentos}

À Fundação de Amparo a Pesquisa do Estado de Mato Grosso (FAPEMAT) pelo investimento na pesquisa.
Este estudo teve a aprovação do Comitê de Ética em Pesquisa Animal (CEPA-UFMT).

\section{Referências}

BUCHANAN, J. W.; BÜCHELER, J. Vertebral scale system to measure canine heart size in radiographs. Journal of the American Veterinary Medical Association, New York, v. 206, n. 2, p. 194-199, 1995.

CARDOSO, M. J. L.; CALUDINO. J. L.; MELUSSI. M. Measurement of heart size by VSH (vertebral heart size) method in healthy american pit bull terrier. Ciência Rural, Santa Maria, v. 41, n. 1, p. 127-131, 2011.

CONSTANTINESCU, G. M. Anatomia clínica de pequenos animais. Rio de Janeiro: Guanabara Koogan, 2005. $355 \mathrm{p}$.

FELIPPE, P. A. N. Eletrocardiografia. In: CUBAS, Z. S.; SILVA, J. C. R.; CATÃO-DIAS, J. L. Tratado de animais selvagens - medicina veterinária. São Paulo: Roca, 2007. p. 920-929.

FONSECA, P. A. C. B. C. Radiologia. In: CUBAS, S. Z; SILVA, J. C. R.; CATÃO-DIAS, J. L. Tratado de animais selvagens - medicina veterinária. São Paulo: Editora Roca Ltda, 2007. p. 896-919.

FRANDSON, R. D. Anatomia e fisiologia dos animais da fazenda. 6. ed. Rio de Janeiro: Guanabara Koogan, 2005. $454 \mathrm{p}$.

GREGORES, G. B. Topografia vértebro-medular e anestesia espinhal em quati (Nasua nasua). 2006. Dissertação (Mestrado em Anatomia dos Animais Domésticos e Silvestres) - Faculdade de Medicina Veterinária e Zootecnia. Universidade de São Paulo. Disponível em: <http://www.teses.usp.br/teses/ disponiveis/10/10132/tde-28022007-150039/>. Acesso em: 26 jun. 2013.

HANSSON, K.; HAGGSTROM, J.; KVART, C.; LORD, P. Interobserver variability of vertebral heart size measurements in dogs with normal and enlarged hearts. Veterinary Radiology \& Ultrasound, Virgínia, v. 46, n. 2, p. 122-130, 2005.

HERRTAGE, M. E.; DENNIS, R. El Tórax. In: LEE, R. Manual de diagnóstico por imagen en pequeños animals. Madrid: Harcurt Brace de Espana, 1999. cap. 3, p. 47-78.

KIDO, S.; IKEZOE, J.; TAKEUCHI, N.; KONDOH, H.; JOHKOH, T.; KOHNO, N.; TOMIYAMA, N.; YAMAGAMI, H.; NAITO, H.; ARISAWA, J.; MORIMOTO, S.; KOZUKA, T. Interpretation of subtle 
interstitial lung abnormalities: conventional versus filmdigitized radiography. Radiology, v. 192, n. 1, p. 171176, 1994.

LAMB, C. R.; TYLER, M.; BOSWOOD, A.; SKELLY, B. J.; CAIN, M. Assessment of the value of the vertebral heart scale in the radiographic diagnosis of cardiac disease in dogs. Veterinary Record, London, v. 146, n. 24, p. 687-690, 2000.

LAMB, C. R.; WIKELEY, H.; BOSWOOD, A.; PFEIFFER, D. U. Use of breed-specific ranges for the vertebral scale as an aid to the radiographic diagnosis of cardiac diseases in dogs. Journal of the American Veterinary Medical Association, New York, v. 230, n. 12, p. 1870-1876. 2001.

MARIN, L. M.; BROWN, J.; MCBRIEN, C.; BAUMWART, R.; SAMII, V. F.; COUTO, C. G. Vertebral heart size in retired racing greyhounds. Veterinary Radiology and Ultrassound, Virgínia, v. 48, n. 4, p. 332334. 2007.

NAKAYAMA, H.; NAKAYAMA, T.; HAMLIN, R. L. Correlation of cardiac enlargement as assessed by vertebral heart size and echocardiographic and electrocardiographic findings in dogs with evolving cardiomegaly due to rapid ventricular pacing. Journal of Veternary Internal Medicine, United Kingdom, v. 15, n. 2, p. 217-221, 2001.

PATTON, S.; FAULKNER, C. T. Prevalence of Dirofilaria immitis and Dipetalonema reconditum infection in dogs: 805 cases (1980-1989). Journal of the American Veterinary Medical Association, New York, 200:1533-1534, 1992.

PINTO, F. A. C. B. C.; IWASAKI, M. Avaliação radiográfica da silhueta cardíaca pelo método de mensuração vhs (Vertebral Heart Size) em cães da raça poodle clinicamente normais. Brazilian Journal of Veterinary Research and Animal Science, São Paulo, v. 41, n. 4, p. 261-267, 2004.

RAMIREZ, E. Y. Manual Clínico de cardiologia básica en el perro y el gato. Zaragoza: Servet, 2005. 288 p.
ROOT, C. R.; BAHR, R. J. The heart and great vessels. In: THRALL, D. E. Textbook of veterinary diagnostic radiology. 3. ed. Philadelphia: W.B.Saunders Company, 1998. cap. 30, p. 335-352.

SILVA, R. A. M. S.; SOUZA-LIMA, E. S. Alterações hematológicas e bioquímicas em Quatis (Nasua nasua) naturalmente infectados por Trypanosoma evansi no Pantanal, MS. Corumbá: Embrapa Pantanal, 2004. (Circular técnica, v. 55).

SISSON, D. D.; THOMAS, W. P.; BONAGURA, J. Cardiopatia congênita. In: ETTINGER, S. J.; FELDMANN, E. C. Tratado de medicina interna veterinária. 5. ed. São Paulo: Guanabara Koogan, v. 1, 2004. p. 780-795.

SISSON, R. G.; GROSSMAN, J. D. Anatomia dos animais domésticos. 5. ed. São Paulo: Nova Guanabara, 2000. 2 v.

STEPIEN, R. L.; BENSON, K. G.; FORREST, L. J. Radiographic measurement of cardiac size in normal ferrets. Veterinary Radiology and Ultrasound, Virgínia, v. 40 , n. 6 , p. 606-610, 1999.

SUTER, P. F. Tórax. In: TICER, J. W. Técnicas radiológicas na prática veterinária. 2. ed. São Paulo: Roca, 1987. cap. 15. p. 298-327.

TEIXEIRA, R. H. F.; AMBRÓSIO, S. R. Carnívora, Procyonidae (mão pelada, quati, jupará). In: CUBA, Z. S.; SILVA, J. C. R.; CATÃO-DIAS, J. L. (Ed.). Tratado de animais selvagens. São Paulo: Editora Roca, 2007. p. 571-573.

THRALL, D. E. Textbook of veterinary diagnostic radiology. 3. ed. Philadelphia: W.B.Saunders Company, 1998.

VAN DEN BROEK, A. H. M.; DARKE, P. G. G. Cardiac mensuraments on thoracic radiographs of cats. Journal of Small Animal Practice, Oxford, v. 28, n. 7, p. 125-135, 1987.

ZANDVLIET, M. M. J. M. Electrocardiography in psittacine birds and ferrets. Seminars in Avian and Exotic Pet Medicine, Barcelona, v. 14, n. 1, p. 34-51, 2005. 
